\title{
Impact des pratiques de traite des éleveurs sur la qualité sanitaire du lait de chamelle en Mauritanie
}

\author{
I. Tourette ${ }^{1}$ S. Messad ${ }^{2}$ B. Faye ${ }^{2 *}$
}

\begin{abstract}
Mots-clés
Camelus dromedarius -

Lait de chamelle - Qualité -

Traite manuelle - Mauritanie.
\end{abstract}

\begin{abstract}
Résumé
L'étude a eu pour objectif d'évaluer les relations entre les pratiques de traite et la qualité sanitaire du lait cru de chamelle. Elle a été menée de mai à août 2001 en Mauritanie, dans les régions du Trarza et du Brakna, à la frontière avec le Sénégal. L'amélioration de la qualité sanitaire du lait représente un enjeu commercial certain puisque les Mauritaniens sont de grands consommateurs de lait local et que la laiterie "Laitière de Mauritanie » souhaite produire du lait stérilisé longue conservation. Un échantillon des fournisseurs de la Laitière de Mauritanie a été enquêté et le lait de leurs chamelles prélevé afin de le soumettre à une série d'analyses bactériologiques : dénombrement des flores aérobie mésophile et coliforme, réduction du bleu de méthylène et test de la résazurine, et titrage de l'acidité Dornic. Les producteurs qui ont eu les moins bonnes pratiques de traite ont eu les plus mauvais résultats en termes de qualité sanitaire du lait de traite. En moyenne, le lait a été peu contaminé juste après la traite. La moyenne des dénombrements a été de 1,6 × $10^{6}$ germes aérobies mésophiles $/ \mathrm{ml}$ et de 3,5 × $10^{4}$ germes coliformes $/ \mathrm{ml}$. La qualité sanitaire du lait acheminé du lieu de production au centre de collecte en plus de trois heures et demi s'est détériorée significativement. Le titrage de l'acidité Dornic a été indépendant des résultats des autres analyses réalisées. Cette étude a confirmé l'intérêt de mettre en place une formation des éleveurs à l'hygiène de la traite afin d'obtenir un lait de bonne qualité sanitaire, organoleptique et nutritionnelle.
\end{abstract}

\section{INTRODUCTION}

La Mauritanie est le pays d'Afrique de l'Ouest où la population caméline est la plus importante. L'effectif estimé serait supérieur à un million de têtes (5). L'élevage camélin y est majoritairement de type semi-transhumant, notamment dans le sud du pays, et la vocation de cette activité est la production de lait et de viande. Depuis une dizaine d'années, le lait de chamelle est commercialisé dans les grandes villes, en particulier à Nouakchott, après transformation. La « Laitière de Mauritanie », principale laiterie installée à Nouakchott, transforme entre 8000 et 11000 litres de lait par jour (2). L'enjeu économique de cette production dans un pays à forte tradition laitière est évident. Par ailleurs, l'attention portée à la qualité sanitaire des produits prend de plus en plus d'importance dans les pays du Sud, à l'instar des pays du Nord. Or, la contamination microbienne peut rendre le lait, produit hautement péris-

1. Association des producteurs de la laiterie Tiviski, BP 2069, Nouakchott, Mauritanie

2. Cirad-emvt, TA 30/A, Campus international de Baillarguet, 34398 Montpeliier Cedex, France

* Auteur pour la correspondance sable, impropre à la consommation humaine, suite à une altération organoleptique, ou présenter un danger pour la santé publique (présence de germes pathogènes, toxines, etc.). Le lait de chamelle, en dépit de ses qualités naturelles (c'est un produit riche en lysozymes aux propriétés bactéricides, par exemple), n'échappe pas aux problèmes de contamination. En outre, la production laitière n'est pas régulière tout au long de l'année et les pics de production ne correspondent pas aux pics de consommation $(1,6)$. Transformer une partie du lait en lait stérilisé Uht est un des moyens envisagés pour ajuster la production à la demande. Cependant, produire du lait stérilisé impose de disposer d'une matière première de bonne qualité microbiologique. Enfin, les données disponibles sur le niveau de contamination du lait de chamelle sont rares. La présente étude se focalise sur les relations entre pratiques de traite et qualité microbiologique du lait de chamelle.

\section{MATERIEL ET METHODES}

\section{Echantillonnage}

L'étude a été menée sur un échantillon de 177 chamelles en lactation appartenant à 39 producteurs tirés au hasard parmi 
les fournisseurs de la Laitière de Mauritanie. Dans chaque troupeau, les cinq premières chamelles traites ont été prélevées. Chaque producteur enquêté a été soumis à un questionnaire portant sur ses pratiques d'élevage et de traite. La traite de son troupeau a été suivie et répertoriée. Pour chacune des 177 chamelles, trois tubes de $15 \mathrm{ml}$ ont servi à prélever des échantillons dans le récipient de traite contenant son lait dès la fin de la traite, deux tubes ont été plongés dans un mélange eau-glace et le dernier a été conservé à température ambiante jusqu'à réception du lait au centre de collecte. Les échantillons ont alors été stockés dans un réfrigérateur à $4{ }^{\circ} \mathrm{C}$ et analysés le lendemain matin. Chaque chamelle prélevée a été soumise à un dépistage de mammite à l'aide de l'épreuve de Whiteside (3).

\section{Analyses de laboratoire}

Les analyses réalisées sur le lait ont été les suivantes :

- le dénombrement de la flore aérobie mésophile totale (Famt) en milieu gélosé pour dénombrement à $30^{\circ} \mathrm{C}$ pendant $72 \mathrm{~h}$. Les résultats ont été donnés en germes par millilitre plutôt qu'en unités formant colonies (Ufc) afin de faciliter les comparaisons avec les données de la littérature, essentiellement exprimées en germes par millilitre ;

- le dénombrement de la flore coliforme totale, sur milieu désoxycholate à $30^{\circ} \mathrm{C}$ pendant $24 \mathrm{~h}$;

- le titrage de l'acidité Dornic ;

- la réduction de la résazurine (notes de 0 à 6 selon les couleurs après réaction) ;

- le temps de réduction du bleu de méthylène (analyse réalisée sur les échantillons de lait réfrigérés immédiatement après la traite et ceux réfrigérés après un délai correspondant au temps de transport). Généralement un temps de réduction supérieur à $4 \mathrm{~h}$ a été considéré comme suffisant pour attester de la bonne qualité du lait.

Les méthodes utilisées ont été celles décrites par Guiraud (10).

\section{Analyses statistiques}

L'objectif ayant été de disposer de profils bactériologiques des échantillons de lait construit à partir de plusieurs critères et de mettre en relation ces profils avec un ensemble de pratiques de traite, les analyses statistiques se sont basées sur les méthodes multivariées $(12,18)$ : l'analyse des correspondances multiples et classification ascendante hiérarchique avec critère de Ward pour établir une typologie des pratiques de traite $(7,13)$; et l'analyse factorielle discriminante (13) et un test de Monte-Carlo (14) pour déceler les relations entre pratiques de traite et profils bactériologiques des laits.

\section{RESULTATS}

Les exploitations laitières situées à la périphérie de Nouakchott ont été majoritairement de type familial : 50 p. 100 d'entre elles ont eu une production laitière quotidienne inférieure à 501 et 75 p. 100 ont eu moins de 100 chamelles. Le rayon de collecte de la laiterie a été de $60 \mathrm{~km}$. Le délai moyen entre la traite et la réception du lait au centre de collecte a été situé dans l'intervalle [ 2 h $30 ; 3$ h 10]. Parmi les chamelles prélevées, 50 p. 100 ont produit moins de 51 par jour, 80 p. 100 ont eu au plus trois lactations et 25 p. 100 ont eu un résultat positif à l'épreuve de Whiteside.

\section{Quelques ordres de grandeur des fréquences de pratiques de traite}

Parmi les producteurs enquêtés, 50 p. 100 trayaient dans un endroit propre (peu souillé par les fèces des animaux) et la même proportion utilisait un récipient propre pour recueillir le lait de traite. Environ 40 p. 100 filtraient le lait avec un tissu de type moustiquaire qui permettait d'éliminer les plus grosses particules (ectoparasites, brindilles, etc.), 40 p. 100 travaillaient avec une tenue jugée sale et 75 p. 100 ne se lavaient pas les mains avant de traire les chamelles. Seuls 7 p. 100 se lavaient les mains avec du savon ; les mains n'étaient jamais séchées.

\section{Résultats des tests bactériologiques et physiques (degrés Dornic)}

Le lait a globalement été peu contaminé lorsqu'il a été prélevé juste après la traite (tableau I). Le temps de réduction du bleu de méthylène après délai de réception a été d'autant plus court que le transport a été long : pour les laits reçus avec un délai supérieur à 3 h 35 (soit 25 p. 100 des laits), la diminution du temps de réduction du bleu de méthylène a été significativement plus importante ( $\mathrm{p}<0,05)$. Les éleveurs ont ainsi été pénalisés quand le délai de réception a été trop long : leur lait n'a pas été réfrigéré pendant le temps de latence qui caractérise la croissance de toute population bactérienne (16) et la contamination microbiologique du lait a donc été plus importante à la réception qu'à la production.

Enfin, les résultats aux tests suivants ont été très corrélés : temps de réduction du bleu de méthylène, réduction de la résazurine et dénombrement de la Famt. En revanche, le titrage de l'acidité Dornic a donné des résultats indépendants des autres résultats bactériologiques.

\section{Typologie des pratiques de traite}

Après analyse des pratiques (analyse des correspondances multiples suivie d'une classification), les producteurs ont été regroupés selon trois types de pratiques résumés dans le tableau II. Globalement, la manière de traire du type I a été qualifiée de "propre », celle du type II de « correcte » et celle du type III de « sale ». De plus, la majorité des producteurs du type I ont été enquêtés pendant la saison des pluies, dans une zone où les points d'eau ont été peu nombreux et souvent payants. Les chamelles ont été plus fréquemment sales puisqu'elles se couchaient dans les flaques et l'eau ménagère utilisée pouvait provenir des mares temporaires. Le type I a eu des pratiques de traite bonnes mais des chamelles sales et le type III des pratiques de traite mauvaises mais des chamelles propres. Seule une variable utilisée pour établir la classification ne figure pas dans le tableau II car elle n'était pas significative pour la typologie : celle de l'ancienneté des éleveurs, c'est-à-dire du nombre de mois depuis lequel l'éleveur a vendu du lait à la laiterie.

\section{Relations entre pratiques de traite et qualité hygiénique du lait}

L'analyse factorielle discriminante a permis d'identifier les paramètres les plus influents pour la constitution des types précédemment décrits. Les éleveurs de type I (traite propre) ont produit du lait dont les résultats bactériologiques ont été bons. La qualité du lait des producteurs de type II (traite correcte) a été très bonne. Celle du type III (traite sale) a été mauvaise (tableau III).

Les producteurs de type I ont eu des résultats inférieurs à ceux de type II en termes de qualité hygiénique du lait parce qu'ils ont fourni un lait plus contaminé en Famt. Rappelons que ceux-ci ont été enquêtés au cours de la saison des pluies. Il est possible qu'ils aient été pénalisés par un environnement boueux rendant les chamelles plus sales qu'en saison sèche. Il est en effet difficile de trouver de l'eau propre car l'eau des forages est souvent payante dans cette zone. Les résultats du type III confirment l'intérêt de maintenir une bonne hygiène au moment de la traite. 


\section{Tableau I}

Moyennes des résultats bactériologiques et physiques des laits analysés

\begin{tabular}{|c|c|c|c|}
\hline Analyses & Moyenne & Intervalle de confiance à $95 \%$ & Signification et normes \\
\hline Dénombrement Famt ${ }^{1}$ (germes/ml) & $1,6 \times 10^{6}$ & {$\left[0-3,5 \times 10^{6}\right]$} & Norme française $<5 \times 10^{5}$ \\
\hline Dénombrement des coliformes (germes/ml) & $3,5 \times 10^{4}$ & {$\left[175-7 \times 10^{4}\right]$} & $<100$ germes/ml \\
\hline Réduction de la résazurine & 3 & {$[2-3]$} & $\begin{array}{l}0,1 \text { (et } 2 \text { ) : lait contaminé } \\
\text { (2) } 3 \text { et } 4: \text { peu contaminé } \\
\text { (4) } 5 \text { et } 6: \text { bonne qualité }\end{array}$ \\
\hline Acidité Dornic ( ${ }^{\circ}$ Dornic) & $19,3^{\circ} \mathrm{D}$ & {$[19,0-19,7]$} & $\begin{array}{l}\text { Norme française : } 14 \text { à } 18^{\circ} \mathrm{D} \\
\text { Lait contaminé si }>20^{\circ} \mathrm{D}\end{array}$ \\
\hline $\begin{array}{l}\text { Temps de réduction du bleu de méthylène } \\
\text { (heures) }\end{array}$ & $4 \mathrm{~h} 20$ & {$[4$ h $10-4$ h 30$]$} & $\begin{array}{l}<2 \mathrm{~h} \text { : lait contaminé } \\
2 \mathrm{à} 4 \mathrm{~h}: \text { peu contaminé } \\
>4 \mathrm{~h} \text { : bonne qualité }\end{array}$ \\
\hline $\begin{array}{l}\text { Temps de réduction du bleu de méthylène } \\
\text { après délai de transport (heures) }\end{array}$ & 3 h 25 & {$[3$ h $10-3$ h 40$]$} & Idem \\
\hline
\end{tabular}

${ }^{1}$ Flore aérobie mésophile totale

\section{Tableau II}

Typologie des pratiques de traite

\begin{tabular}{|c|c|c|c|}
\hline & Type I & Type II & Type III \\
\hline Propreté des mamelles & Insuffisante & & Correcte \\
\hline Propreté du lieu de traite & & Insuffisante & Correcte \\
\hline Propreté du récipient de traite & Propre à très propre & Correcte & Insuffisante \\
\hline Propreté de la tenue du trayeur & & Correcte & Insuffisante \\
\hline Lavage des mains avant la traite & Oui & Non & Non \\
\hline Emploi de trayeurs & & Non & \\
\hline Filtrage du lait & Oui & & \\
\hline Traite & Propre & Correcte & Sale \\
\hline Nombre de producteurs & 14 & 10 & 15 \\
\hline
\end{tabular}

\section{Tableau III}

Résumé des liens entre types de pratiques de traite et qualité microbiologique du lait

\begin{tabular}{|c|c|c|c|}
\hline Analyse & Type I & Type II & Type III \\
\hline Temps de réduction du bleu de méthylène & $>5 \mathrm{~h} 00$ & $>4 \mathrm{~h} 00$ & $\begin{array}{c}<4 \text { h } 00 \text { pour } 25 \% \\
\text { des échantillons }\end{array}$ \\
\hline Contamination en Famt ${ }^{1}$ (germes/ml) & $1,7 \times 10^{4}$ à $1,7 \times 10^{4}$ & 0 à $1,7 \times 10^{4}$ & $7,9 \times 10^{4}$ à $1,5 \times 10^{8}$ \\
\hline $\begin{array}{l}\text { Contamination en coliformes } \\
\text { (germes } / \mathrm{ml} \text { ) }\end{array}$ & $\begin{array}{l}\text { Absence d'échantillons } \\
\text { très contaminés }\end{array}$ & $\begin{array}{l}0 \text { à } 5 \times 10^{3} \\
\text { Contamination } \\
\text { importante }\end{array}$ & $\begin{array}{l}5 \times 10^{3} \text { à } 1,3 \times 10^{6} \\
\text { Contamination } \\
\text { très importante }\end{array}$ \\
\hline
\end{tabular}

Les variables microbiologiques ont été les plus liées aux types d'éleveurs ; elles ont été tirées d'une analyse factorielle discriminante

${ }^{1}$ Flore aérobie mésophile totale 


\section{DISCUSSION}

Les bons résultats sur le plan bactériologique ont été observés logiquement chez les producteurs ayant eu les pratiques de traite les plus propres. Les auteurs n'ont pas pu mettre en évidence de différence entre les trois types de producteurs quant aux dénombrement de la flore coliforme, réduction de la résazurine et titrage de l'acidité Dornic. D'après les données du tableau I, il apparaît que le lait a globalement été peu contaminé lorsqu'il a été prélevé juste après la traite.

\section{Limites de la méthode}

Les analyses bactériologiques ont été réalisées dans des conditions difficiles qui pouvaient être à l'origine de légères différences dans les résultats microbiologiques. Ces résultats sont à prendre comme des ordres de grandeur plutôt que comme des résultats précis. Les échantillons de lait ayant été réfrigérés, le temps de réduction du bleu de méthylène peut en être augmenté (10). Cela expliquerait l'importance des temps de réduction obtenus.

L'évaluation de l'état de propreté des chamelles (tenue, lieu et récipient) a été réalisée de manière subjective par un seul et même enquêteur. Il eut été judicieux de travailler avec une grille de notation pour les différents paramètres notés afin d'objectiver l'état de propreté. Cependant, l'unicité du notateur devrait limiter ce biais d'observation (9).

Enfin, des analyses de l'eau utilisée dans les différentes exploitations auraient permis de vérifier le rôle de la qualité de l'eau comme facteur de confusion. En effet, une eau insalubre détériore la qualité hygiénique du lait en contaminant le matériel de traite. Des analyses bactériologiques de l'eau ont été réalisées dans le cadre d'une étude menée au Tchad en 1995 sur la qualité sanitaire de lait cru et de produits laitiers traditionnels. Les eaux de ménage utilisées par les Arabes transhumants ont des contaminations bactériennes moyennes de $1,7 \times 10^{5}$ germes $/ \mathrm{ml}$ pour la flore totale et de $5 \times 10^{2}$ germes $/ \mathrm{ml}$ pour les coliformes (16), nombres se situant dans l'intervalle de confiance des moyennes de contaminations des normes françaises pour le lait (tableau I).

\section{Comparaison des résultats}

La norme française pour l'acidité Dornic du lait cru est donnée pour du lait de vache dont le $\mathrm{pH}$ est compris entre 6,6 et 6,8 (11). Or le pH du lait de chamelle est compris entre 6,5 et 6,7 (8). Ceci peut expliquer l'importance du nombre de degrés Dornic obtenu en moyenne pour ces laits de chamelle. Il n'y a cependant pas de relation directe entre $\mathrm{pH}$ et acidité Dornic en raison du pouvoir tampon du lait.

Une étude sur la qualité sanitaire du lait de chamelle a été réalisée en Egypte en mars 2000 sur un échantillon de 50 chamelles. La contamination moyenne en Famt a été de 4,3 x $10^{4}$ germes $/ \mathrm{ml}$ et celle en coliformes de $2,9 \times 10^{2}$ germes/ml (15). Les échantillons prélevés dans le cadre de la présente étude ont été cent fois plus contaminés que les échantillons égyptiens. Cette différence pouvait provenir des conditions de laboratoire difficiles mais aussi de réelles différences de qualité sanitaire des laits analysés. Les pratiques de traite des éleveurs égyptiens ne sont pas détaillées. Peutêtre observent-ils de meilleures pratiques de traite et d'élevage. Quoi qu'il en soit, la rareté des résultats de ce type ne permet pas de comparer davantage les résultats obtenus ici.

\section{Perspectives}

La population de producteurs enquêtés faisait partie d'une association de producteurs dont le but était d'améliorer la productivité laitière des animaux ainsi que la qualité sanitaire du lait. Il serait intéressant d'entreprendre une nouvelle étude de ce genre dans quelque temps afin d'évaluer l'impact des formations dispensées par l'asso- ciation sur la qualité du lait. Cette nouvelle étude devra adopter des conditions d'analyse plus rigoureuses et une grille de notation pour attribuer des notes de propreté objectives. Des analyses bactériologiques de l'eau permettront de confirmer son rôle en tant que point critique (ou non). D'autres méthodes d'évaluation de la contamination du lait pourraient être utilisées ; par exemple, le titrage de l'acidité Dornic pourrait ne pas être pris en compte.

\section{CONCLUSION}

La qualité hygiénique du lait de chamelle livré à la laiterie de Nouakchott a été parfois insuffisante sans être catastrophique. Cependant, quelques recommandations (ci-après) peuvent être faites pour obtenir un meilleur résultat.

Pour ce qui concerne le milieu, la traite s'effectuera dans un endroit propre en séparant les aires de traite et d'alimentation. Les chamelles resteront propres par un nettoyage régulier des chmels (protège-mamelles qui empêchent le petit de téter au pâturage). Concernant le matériel, il s'agira de nettoyer et de désinfecter le récipient de traite avant et après la traite (à l'intérieur et à l'extérieur), d'utiliser des cordes propres pour attacher le petit à la mère et entraver les chamelles, et de n'utiliser de filtre que s'il est parfaitement propre. Pour la main d'œuvre, il importera de se laver les mains avec du savon avant de traire, de les sécher et de réserver une tenue propre pour la traite. A propos de la méthode, il faudra nettoyer les mamelles avec un tissu propre et sec avant de traire et ne pas poser le matériel au sol (cordes, récipient de traite). Pour la matière première, il sera important de ne pas mélanger le lait de mammite avec le lait des chamelles saines, de traiter les mammites, de respecter les temps d'attente des antibiotiques et anthelminthiques, d'éliminer le premier jet de chaque quartier, d'entreposer le bidon de lait à l'ombre, voire dans un trou humidifié et, enfin, de diminuer au maximum le délai entre traite et réception du lait au centre de collecte (optimiser les circuits de collecte).

La transformation du lait en lait stérilisé permet de mieux gérer les pics de production qui ne sont pas toujours synchrones des pics de consommation. Pour stériliser du lait, il faut pouvoir, en premier lieu, produire du lait de bonne qualité hygiénique. Cela demande un effort de travail tout à fait envisageable. Si, dans le même contexte que cette étude, on peut obtenir que les producteurs se lavent les mains avant de traire, portent une tenue propre, utilisent un récipient et un lieu de traite propres, disposent d'une eau potable et d'un circuit de collecte efficace, alors la qualité microbiologique du lait permettra sans aucun doute de produire du lait stérilisé. Pour cela, il faut inciter les éleveurs à améliorer l'hygiène de la traite (paiement du lait à la qualité), les aider à diagnostiquer et traiter les mammites, les inciter à éliminer les laits de mammites, ajouter des puits aux endroits stratégiques et judicieusement choisis et concevoir des circuits de collecte courts.

\section{Remerciements}

Nous tenons à remercier Mme Nancy Abeiderrhamane, fondatrice de la Laitière de Mauritanie, pour son accueil, ainsi que Yacoub Cissoko et Abdellahi Salem Ould Lellah, pour leur aide précieuse.

\section{BIBLIOGRAPHIE}

1. ABEIDERRHAMANE N., 1994. La pasteurisation du lait de chamelle : une expérience en Mauritanie. In : Bonnet P., ed., Actes colloque "Dromadaires et chameaux, animaux laitiers ", Nouakchott, Mauritanie, 26-24 octobre 1994. Montpellier, France, Cirad-emvt, p. 213-220.

2. ABEIDERRHAMANE N., 1998. Collecte, transformation et pasteurisation du lait pour I'approvisionnement de Nouakchott : I'expérience de la Laitière de Mauritanie. In : Actes atelier international « Marchés urbains et développement laitier en Afrique subsaharienne », 9-10 septembre 1998. Montpellier, France, Cirad-emvt, p. 157-167. 
3. ABDURAHMAN O.A.SH., 1994. Detection of subclinical mastitis in camels: relationship between udder infection and inflammatory indicators in milkIn : Bonnet P., ed., Actes colloque "Dromadaires et chameaux, animaux laitiers », Nouakchott, Mauritanie, 26-24 octobre 1994. Montpellier, France, Cirad-emvt, p. 31-34.

4. BOUYER J., HEMON D., CORDIER S., DERRIENIC F., STUCKER I., STENGEL B., CLAVEL J., 1995. Epidémiologie, principes et méthodes quantitatives. Paris, France, Inserm, 498 p.

5. DEVEY-MALU MALU M., MASSON-BOISRIVEAU J., RICHARD S., 1998. Mauritanie, marchés tropicaux et méditerranéens, hors série. Bordeaux, France, Cifdi, 24 p.

6. DIOP D., 1994. Production du lait de chamelle (Camelus dromedarius) en Mauritanie : étude technico-économique. Thèse vét., université de Sidi Thabet, Tunisie, 86 p.

7. ESCOFIER B., PAGES J., 1998. Analyses factorielles simples et multiples : objectifs, méthodes et interprétation, $3^{\mathrm{e}}$ edn. Paris, France, Dunod, p. 95-121.

8. FARAH Z., 1993. Composition and characteristics of camel milk. J. Dairy Res., 6: 603-623.

9. FAYE B., CALAVAS D., ROSNER G., 1994. La fiabilité des données dans les enquêtes d'écopathologie. Revue sci. tech. Off. int. Epizoot., 13 : $651-664$

10. GUIRAUD J.-P., 1998. Microbiologie alimentaire. Paris, France, Dunod, p. 652.
11. HARPER, HALL W.J., 1976. Dairy technology and engineering. Westport, CT, USA, AVI Publishing.

12. IHAKA R., GENTLEMAN R., 1996. Language and environment for statistical computing and graphics. J. comput. grap. Stat., 5: 299-314.

13. LEBART L., MORINEAU A., PIRON M., 1995. Statistique exploratoire multidimensionnelle. Paris, France, Dunod, p. 438.

14. MANLY BRYAN J.-F., 1997. Randomization, bootstrap and MonteCarlo methods in biology, 2nd Edn. London, UK, Chapman and Hall, $376 \mathrm{p}$.

15. MOUSTAFA S.I., AHMED A.H., SAAD N.M., MAHMOUD Y.H., 2000. Quality evaluation of camels' milk in new valley governorate, Egypt. J. Agric. Res., 78: 241-248.

16. PISSANG TCHANGAI D., 1998. Evaluation de la qualité du lait et des produits laitiers dans les systèmes traditionnels de transformation au Tchad. In : Actes atelier international "Marchés urbains et développement laitier en Afrique subsaharienne », 9-10 septembre 1998. Montpellier, France, Cirad-emvt, p. 125-133.

17. ROZIER J., CARLIER V., BOLNOT F., 1985. Bases microbiologiques de I'hygiène des aliments. Paris, France, Sepaic, 230 p.

18. THIOULOUSE J., CHESSEL D., DOLEDEC S., OLIVIER J.-M., 1997. ADE-4: a multivariate analysis and graphical display software. Stat. Comput., 7: 75-83.

Reçu le 17.05.2002, accepté le 17.09.2003

\section{Summary}

Tourette I., Messad S., Faye B. Impact of Breeders' Milking Practices on the Sanitary Quality of She-Camel Milk in Mauritania

The aim of the study was to assess the relationships between milking practices and the sanitary quality of raw camel milk. It was conducted from May to August 2001 in Mauritania, in Trarza and Brakna areas along the Senegalese border. Improving the sanitary quality of milk is a commercial challenge since the Mauritanians consume a lot of local milk. In addition, the dairy plant "Laitière de Mauritanie" aims at producing long-life sterilized milk. A number of breeders who supplied milk to this dairy plant were surveyed and samples from their camel milk were collected for bacteriological analyses: aerobic mesophilic and coliform flora count, methylene blue reduction and resazurin tests, and Dornic acidity titration. Milk suppliers with the poorest milking practices had the worst results with regard to the sanitary quality of milk. On average, milk was little contaminated just after milking. Mean counts were $1.6 \times 10^{6}$ aerobic mesophilic germs $/ \mathrm{ml}$ and $3.5 \times 10^{4}$ coliforms $/ \mathrm{ml}$. The sanitary quality of milk decreased significantly when the milk was carried from the production area to the collecting center in more than three and a half hours after milking. Results from Dornic acidity titration were not coherent with those from the other analyses. This study confirms the need to set up a farmer's training program on milking hygiene to produce milk with organoleptic, nutritional and high sanitary quality.

Key words: Camelus dromedarius - Camel milk - Quality Hand milking - Mauritania.

\section{Resumen}

Tourette I., Messad S., Faye B. Impacto de las prácticas de ordeño de los criadores sobre la calidad sanitaria de la leche de la camella en Mauritania

El presente estudio tuvo por objetivo la evaluación de las relaciones entre las prácticas de ordeño y la calidad sanitaria de la leche cruda de camella. Se llevó a cabo entre mayo y agosto 2001 en Mauritania, en las regiones de Trarza y Brakna, en la frontera con Senegal. La mejoría en la calidad sanitaria de la leche representa una importancia comercial cierta, debido a que los Mauritanos son grandes consumidores de leche local y la lechería "Lechería de Mauritania" desea producir leche estéril de conservación prolongada. Una muestra de los provedores de la lechería de Mauritania fue estudiada y se obtuvieron muestras la leche de las camellas, esto con el fin de someterlas a una serie de análisis bacteriológicos: conteo de floras aeróbicas mesófilas y coliforme, reducción de azul de metileno y de la resarusina, título de acidez de Dornic. Los productores que presentaron las prácticas menos buenas de ordeño obtuvieron los peores resultados en términos de calidad sanitaria de la leche de ordeño. En promedio, la leche estuvo poco contaminada justo después del ordeño. El promedio de conteos fue de 1,6 x $10^{6}$ gérmenes aeróbicos mesófilos $/ \mathrm{ml}$ y de $3,5 \times 10^{4}$ gérmenes coliformes $/ \mathrm{ml}$. La calidad sanitaria de la leche conducida desde el sitio de producción al centro de colecta en más de tres horas y media se deterioró significativamente. El título de acidez de Dornic fue independiente de los resultados de los otros tests realizados. Este trabajo confirma el interés de poner en práctica una formación para los criadores sobre la higiene del ordeño con el fin de obtener leche de buena calidad sanitaria, organoléptica y nutricional.

Palabras clave: Camelus dromedarius - Leche de camella Calidad - Ordeno manual - Mauritania. 